\title{
Topological AE(0)-groups
}

\author{
by
}

Alex Chigogidze (Saskatoon, SK)

\begin{abstract}
We investigate topological AE(0)-groups, a class which contains the class of Polish groups as well as the class of all locally compact groups. We establish the existence of a universal $\mathrm{AE}(0)$-group of a given weight as well as the existence of a universal action of an $\mathrm{AE}(0)$-group of a given weight on an $\mathrm{AE}(0)$-space of the same weight. A complete characterization of closed subgroups of powers of the symmetric group $S_{\infty}$ is obtained. It is also shown that every $\mathrm{AE}(0)$-group is Baire isomorphic to a product of Polish groups. These results are obtained by using the spectral descriptions of $\mathrm{AE}(0)$-groups which are presented in Section 3.
\end{abstract}

1. Introduction. One of the main structure theorems for compact groups (see [10, Chapters 6 \& 9]) can be formulated as follows.

Theorem A ([10], Theorem 9.24(ii)). Let $G$ be a connected compact group. Then there exists a continuous homomorphism

$$
p: Z_{0}(G) \times \prod\left\{L_{t}: t \in T\right\} \rightarrow G,
$$

where $Z_{0}(G)$ stands for the identity component of the center of $G$, and $L_{t}$ is a simple, connected and simply connected compact Lie group, $t \in T$, such that $\operatorname{ker}(p)$ is a zero-dimensional central subgroup of $Z_{0}(G) \times \prod\left\{L_{t}: t \in T\right\}$.

The above statement clearly shows that the classes of zero-dimensional groups, abelian groups and simple, simply connected Lie groups play a central role in the general theory of compact groups. Recently these classes of topological groups have been studied from the point of view of absolute extensors in dimension $n$ (see [4] for a comprehensive introduction into the theory of absolute extensors in dimension $n$ ). The following three statements show that such an approach is quite effective.

2000 Mathematics Subject Classification: Primary 22A05; Secondary 22F05.

Key words and phrases: topological AE(0)-group, Polish group, inverse spectrum.

The author was partially supported by an NSERC research grant. 
TheOREM B ([2]). The following conditions are equivalent for a zerodimensional topological group $G$ :

(a) $G$ is topologically equivalent to the product $\left(\mathbb{Z}_{2}\right)^{\tau} \times \mathbb{Z}^{\kappa}$.

(b) $G$ is an $\mathrm{AE}(0)$-space.

Theorem C ([6], Theorem E). The following conditions are equivalent for a compact abelian group $G$ :

(a) $G$ is a torus group (both in topological and algebraic senses).

(b) $G$ is an $\mathrm{AE}(1)$-compactum.

TheOREM D ([5], Corollary 1). The following conditions are equivalent for a non-trivial compact group $G$ :

(a) $G$ is a simple, connected and simply connected Lie group.

(b) $G$ is an $\mathrm{AE}(2)$-group with $\pi_{3}(G)=\mathbb{Z}$.

The full classification problem for non-abelian (see Theorem C) and for non-simply connected compact AE(1)-groups remains open. On the other hand, the following two statements provide a complete classification of simply connected compact $\mathrm{AE}(1)$-groups.

Theorem E ([5], Theorem C). The following conditions are equivalent for a compact group $G$ :

(a) $G$ is a simply connected $\mathrm{AE}(1)$-compactum.

(b) $G$ is an $\mathrm{AE}(2)$-compactum.

(c) $G$ is an $\mathrm{AE}(3)$-compactum.

(d) $G$ is a product of simple, connected and simply connected compact Lie groups.

Theorem F ([5], Corollary 2). There is no non-trivial compact $\mathrm{AE}(4)$ group.

We complete this brief survey by pointing out that, as shown by the following statement, for locally compact groups the restriction of being an $\mathrm{AE}(0)$-group is purely formal.

Theorem G (Pontryagin-Haydon, [14], [8]; [4]). Every locally compact group is an $\mathrm{AE}(0)$-space.

Below we study $\mathrm{AE}(0)$-groups. The class of $\mathrm{AE}(0)$-groups contains the class of all (generally speaking, non-metrizable) locally compact groups (Theorem G) as well as the class of all Polish groups. Actually the class of Polish groups coincides with the class of metrizable $\mathrm{AE}(0)$-groups and forms a foundation of the entire theory of $\mathrm{AE}(0)$-groups. We hope that the results presented in Section 3 do indicate a potential for a non-trivial theory of $\mathrm{AE}(0)$-groups which unifies and generalizes the theories of locally compact 
and Polish groups (thus offering a possible approach to the corresponding question posed in [13]).

In Section 3 we present a spectral characterization of $\mathrm{AE}(0)$-groups in terms of well ordered continuous inverse spectra (Theorem 3.4). This characterization states that a non-metrizable topological group $G$ of weight $\tau$ is a $\mathrm{AE}(0)$-group if and only if it is the limit of a well ordered continuous inverse system $\mathcal{S}_{G}=\left\{G_{\alpha}, p_{\alpha}^{\alpha+1}, \alpha<\tau\right\}$ of length $\tau$, consisting of $\operatorname{AE}(0)$-groups $G_{\alpha}$ and 0-soft limit homomorphisms $p_{\alpha}^{\alpha+1}: G_{\alpha+1} \rightarrow G_{\alpha}, \alpha<\tau$, so that $G_{0}$ is a Polish group and each homomorphism $p_{\alpha}^{\alpha+1}, \alpha<\tau$, has a Polish kernel.

Obviously this result cannot be accepted as the one providing a satisfactory reduction of the non-metrizable case to the Polish case. Of course, everything is fine if the weight of $G$ is $\omega_{1}$-in such a case all $G_{\alpha}$ 's, $\alpha<\omega_{1}$, (and not only the very first one, i.e. $G_{0}$ ) are indeed Polish. But if the weight of $G$ is greater than $\omega_{1}$, then all $G_{\alpha}$ 's with $\alpha \geq \omega_{1}$ are non-metrizable.

In order to achieve our final goal and complete the reduction, we analyze 0-soft homomorphisms with Polish kernels between (generally speaking, non-metrizable) $\mathrm{AE}(0)$-groups. A characterization of such homomorphisms, which is recorded in Proposition 3.6, states that a 0-soft homomorphism $f: G \rightarrow L$ of $\mathrm{AE}(0)$-groups has a Polish kernel if and only if there exists a pullback diagram

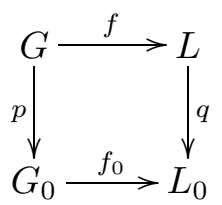

where $G_{0}$ and $L_{0}$ are Polish groups and the homomorphisms $p: G \rightarrow G_{0}$ and $q: L \rightarrow L_{0}$ are 0 -soft. Theorem 3.4 and Proposition 3.6 together complete the required reduction.

Polish groups and their actions have been extensively studied in a variety of directions (ergodic theory, group representations, operator algebras; see [1] for further discussion and references). Some of the central themes of the theory of Polish groups, counterparts of which (for arbitrary AE(0)-groups) are considered below, are the existence of universal groups, the existence of universal actions and characterization of closed subgroups of the symmetric group $S_{\infty}$.

In Section 4 we use the above mentioned spectral characterizations and present extensions of some of these results to $\mathrm{AE}(0)$-groups. We prove the existence of universal $\mathrm{AE}(0)$-groups of a given weight (Proposition 4.1) and the existence of universal actions of $\mathrm{AE}(0)$-groups of a given weight on compact $\mathrm{AE}(0)$-spaces of the same weight (Theorem 4.2).

Theorem 4.4 characterizes $\mathrm{AE}(0)$-groups which are isomorphic to closed subgroups of powers $S_{\infty}^{\tau}$ of the symmetric group $S_{\infty}$, the group of all bijec- 
tions of $\mathbb{N}$ under the relative topology inherited from $\mathbb{N}^{\mathbb{N}}$. This result extends the corresponding observation [1, Theorem 1.5.1] for the group $S_{\infty}$ itself. As a corollary (Corollary 4.5) we note that if a Polish group $G$ can be embedded (as a closed subgroup) into $S_{\infty}^{\tau}$ for some $\tau$, then $G$ can be embedded into $S_{\infty}$ as well. In light of [7] this shows that there exist zero-dimensional Polish groups which cannot be embedded into $S_{\infty}^{\tau}$ as closed subgroups for any cardinal number $\tau$.

Finally we use Theorem 3.4 to prove (Theorem 4.8) that every $\mathrm{AE}(0)$ group is Baire isomorphic to a product of Polish groups. In light of Theorem $\mathrm{G}$ this result appears to be new even for compact groups.

2. Preliminaries. All topological spaces below are assumed to be Tikhonov (i.e. completely regular and Hausdorff) and all maps (except in Subsection 4.3) are continuous. Modified Lebesgue dimension dim is defined in terms of finite functionally open covers so that $\operatorname{dim} X=\operatorname{dim} \beta X$ for any Tikhonov space $X$ ( $\beta X$ denotes the Stone-Cech compactification of $X)$. Definitions of concepts related to inverse spectra can be found in [4]. Finally, $\mathbb{R}$ denotes the real line and $\mathbb{Q}$ stands for the Hilbert cube.

2.1. Definitions of $\mathrm{AE}(n)$-spaces and n-soft maps. A comprehensive introduction to the general theory of $\mathrm{AE}(n)$-spaces and $n$-soft maps can be found in [4]. $C(X)$ denotes the set of all continous real-valued functions defined on $X$.

Definition 2.1. A space $X$ is called an absolute extensor in dimension $n$ (briefly, $\operatorname{AE}(n)$-space), $n=0,1, \ldots$, if for each at most $n$-dimensional space $Z$ and each subspace $Z_{0}$ of $Z$ any map $f: Z_{0} \rightarrow X$ such that $C(f)(C(X)) \subseteq$ $\left\{\varphi \mid Z_{0}: \varphi \in C(Z)\right\}$ can be extended to $Z$.

For compact spaces this definition is equivalent to the standard one.

Proposition 2.2. A compact space $X$ is an $\mathrm{AE}(n)$-space if and only if for each at most $n$-dimensional compactum $Z$ and for each closed subspace $Z_{0}$ of $Z$, any map $f: Z_{0} \rightarrow X$ has an extension to $Z$.

It is known [4, Chapter 6] that the class of metrizable $\mathrm{AE}(0)$-spaces coincides with the class of Polish spaces. Every AE(0)-space is realcompact and has a countable Suslin number.

Definition 2.3. A map $f: X \rightarrow Y$ between $\mathrm{AE}(n)$-spaces is $n$-soft if for each at most $n$-dimensional realcompact space $Z$, for its closed subspace $Z_{0}$, and for any two maps $g: Z_{0} \rightarrow X$ and $h: Z \rightarrow Y$ such that $f \circ g=h \mid Z_{0}$ and $C(g)(C(X)) \subseteq\left\{\varphi \mid Z_{0}: \varphi \in C(Z)\right\}$, there exists a map $k: Z \rightarrow X$ such that $k \mid Z_{0}=g$ and $f \circ k=h$.

It is easy to check that $X$ is an $\mathrm{AE}(n)$-space if and only if the constant map $X \rightarrow\{\mathrm{pt}\}$ is $n$-soft. It is important to note that every 0 -soft map 
between $\mathrm{AE}(0)$-spaces is surjective and open ([4, Lemma 6.1.13 \& Proposition 6.1.26]) and that for surjections between Polish spaces the converse of this fact is also true. Note also that every surjective homomorphism between Polish groups is open and 0-soft.

We say (see [4, Section 6.3]) that a map $f: X \rightarrow Y$ has a Polish kernel if there exists a Polish space $P$ such that $X$ is $C$-embedded in the product $Y \times P$ so that $f$ coincides with the restriction $\pi_{Y} \mid X$ of the projection $\pi_{Y}$ : $Y \times P \rightarrow Y$. Obviously any map between Polish spaces has a Polish kernel.

2.2. Set-theoretical preliminaries. Let $A$ be a partially ordered directed set (i.e. for any two elements $\alpha, \beta \in A$ there exists an element $\gamma \in A$ such that $\gamma \geq \alpha$ and $\gamma \geq \beta$ ). We say that a subset $A_{1} \subseteq A$ of $A$ majorizes another subset $A_{2} \subseteq A$ of $A$ if for each element $\alpha_{2} \in A_{2}$ there exists an element $\alpha_{1} \in A_{1}$ such that $\alpha_{1} \geq \alpha_{2}$. A subset which majorizes $A$ is called cofinal in $A$. A subset of $A$ is said to be a chain if any two of its elements are comparable. The symbol sup $B$, where $B \subseteq A$, denotes the least upper bound of $B$ (if such an element exists in $A$ ). Let now $\tau$ be an infinite cardinal. A subset $B$ of $A$ is said to be $\tau$-closed in $A$ if for each chain $C \subseteq B$ with $|C| \leq \tau$, we have $\sup C \in B$ whenever the element $\sup C$ exists in $A$. Finally, a directed set $A$ is said to be $\tau$-complete if for each chain $B$ in $A$ with $|C| \leq \tau$, the element $\sup C$ exists in $A$.

The standard example of a $\tau$-complete set can be obtained as follows. For an arbitrary set $A$ let $\exp A$ denote, as usual, the collection of all subsets of $A$. There is a natural partial order on $\exp A: A_{1} \geq A_{2}$ if and only if $A_{1} \supseteq A_{2}$. With this partial order $\exp A$ becomes a directed set. If we consider only those subsets of $A$ which have cardinality $\leq \tau$, then the corresponding subcollection of $\exp A$, denoted by $\exp _{\tau} A$, serves as a basic example of a $\tau$-complete set. The proofs of the following statements can be found in [4].

Proposition 2.4. Let $\left\{A_{t}: t \in T\right\}$ be a collection of $\tau$-closed cofinal subsets of a $\tau$-complete set $A$. If $|T| \leq \tau$, then $\bigcap\left\{A_{t}: t \in T\right\}$ is also cofinal (in particular, non-empty) and $\tau$-closed in $A$.

Corollary 2.5. For each subset $B$ with $|B| \leq \tau$ of a $\tau$-complete set $A$ there exists an element $\gamma \in A$ such that $\gamma \geq \beta$ for each $\beta \in B$.

Proposition 2.6. Let $A$ be a $\tau$-complete set, $L \subseteq A^{2}$, and suppose the following three conditions are satisfied:

- Existence: For each $\alpha \in A$ there exists $\beta \in A$ such that $(\alpha, \beta) \in L$.

- Majorization: If $(\alpha, \beta) \in L$ and $\gamma \geq \beta$, then $(\alpha, \gamma) \in L$.

- $\tau$-closedness: Let $\left\{\alpha_{t}: t \in T\right\}$ be a chain in $A$ with $|T| \leq \tau$. If $\left(\alpha_{t}, \beta\right) \in L$ for some $\beta \in A$ and each $t \in T$, then $(\alpha, \beta) \in L$ where $\alpha=$ $\sup \left\{\alpha_{t}: t \in T\right\}$. 
Then the set of all L-reflexive elements of $A$ (an element $\alpha \in A$ is $L$-reflexive if $(\alpha, \alpha) \in L)$ is cofinal and $\tau$-closed in $A$.

2.3. Baire sets and Baire isomorphisms. Recall that elements of the $\sigma$-algebra generated by functionally open subsets of a space $X$ are called Baire sets of $X$. A map $f: X \rightarrow Y$ is a Baire map if the inverse images of Baire sets are Baire sets. A bijection $f: X \rightarrow Y$ is a Baire isomorphism if both $f$ and $f^{-1}$ are Baire maps.

The following statement ([3, Proposition 2.5]) is used in the proof of Theorem 4.8.

Proposition 2.7. Let $f: X \rightarrow Y$ be a 0-soft map of $\mathrm{AE}(0)$-spaces. Then there exists a Baire map $g: Y \rightarrow X$ such that $f \circ g=\operatorname{id}_{Y}$.

If $f$ is a continuous homomorphism of Polish groups, then the existence of such a $g$ has been observed by Dixmier [1, Theorem 1.2.4], [11, Theorem 12.17]).

3. $\mathrm{AE}(0)$-groups and actions of $\mathrm{AE}(0)$-groups - spectral representations. In this section we present spectral characterizations of $\mathrm{AE}(0)$ groups. We also present a spectral description of actions of $\mathrm{AE}(0)$-groups on $\mathrm{AE}(0)$-spaces. An inverse spectrum $\mathcal{S}_{G}=\left\{G_{\alpha}, p_{\alpha}^{\beta}, A\right\}$ is factorizing if for any continuous function $f: \lim \mathcal{S}_{G} \rightarrow \mathbb{R}$ there exist an index $\alpha \in A$ and a continuous function $f_{\alpha}: G_{\alpha} \rightarrow \mathbb{R}$ such that $f=f_{\alpha} \circ p_{\alpha}$.

Proposition 3.1. Each topological $\mathrm{AE}(0)$-group $G$ is topologically and algebraically isomorphic to the limit of a factorizing $\omega$-spectrum $\mathcal{S}_{G}=\left\{G_{\alpha}\right.$, $\left.p_{\alpha}^{\beta}, A\right\}$ consisting of Polish groups $G_{\alpha}, \alpha \in A$, and 0 -soft limit homomorphisms $p_{\alpha}: G \rightarrow G_{\alpha}, \alpha \in A$.

Proof. By [4, Theorem 6.3.2 or Proposition 6.3.5], the space $G$ can be represented as the limit space of a factorizing $\omega$-spectrum $\mathcal{S}=\left\{G_{\alpha}, p_{\alpha}^{\beta}, \widetilde{A}\right\}$ consisting of Polish spaces (i.e. AE(0)-spaces of countable weight) and 0 -soft limit projections. Let us show that this spectrum contains an $\omega$-closed cofinal subspectrum consisting of topological groups and limit projections that are (continuous) homomorphisms.

Let $\mu: G \times G \rightarrow G$ and $\nu: G \rightarrow G$ be the continuous operations of multiplication and inversion coming from the topological group structure of $G$. We apply [4, Theorem 1.3.6] to both $\mu$ and $\nu$. First consider the multiplication. Clearly, $G \times G$ is the limit space of the spectrum

$$
\mathcal{S} \times \mathcal{S}=\left\{G_{\alpha} \times G_{\alpha}, p_{\alpha}^{\beta} \times p_{\alpha}^{\beta}, \widetilde{A}\right\}
$$

All projections of the spectrum $\mathcal{S} \times \mathcal{S}$ are 0 -soft, and hence, by [4, Proposition 6.1.26], open. The Suslin number of the product $G \times G$ is obviously countable (see [4, Proposition 6.1.8]). Consequently, by [4, Proposition 1.3.3], 
the spectrum $\mathcal{S} \times \mathcal{S}$ is factorizing. Next we apply [4, Theorem 1.3.6] to the spectra $\mathcal{S} \times \mathcal{S}, \mathcal{S}$ and to the map $\mu$ between their limit spaces. Then we get an $\omega$-closed cofinal subset $A_{\mu}$ of $\widetilde{A}$ such that for each $\alpha \in A_{\mu}$ there exists a continuous map $\mu_{\alpha}: G_{\alpha} \times G_{\alpha} \rightarrow G_{\alpha}$ such that the diagram

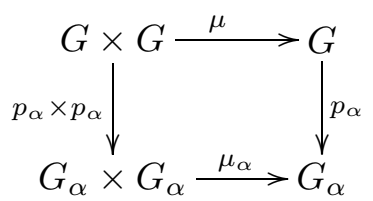

commutes. In other words, $p_{\alpha} \circ \mu=\mu_{\alpha} \circ\left(p_{\alpha} \times p_{\alpha}\right)$ for each $\alpha \in A_{\mu}$.

Next consider the continuous inversion $\nu: G \rightarrow G$. By [4, Theorem 1.3.6], applied to $\nu$ and the spectrum $\mathcal{S}$, there exists an $\omega$-closed cofinal subset $A_{\nu}$ of $\widetilde{A}$ such that for each $\alpha \in A_{\nu}$ there exists a continuous map $\nu_{\alpha}: G_{\alpha} \rightarrow G_{\alpha}$ such that the diagram

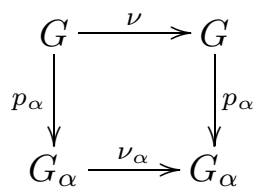

commutes. In other words, $p_{\alpha} \circ \nu=\nu_{\alpha} \circ p_{\alpha}$ for each $\alpha \in A_{\nu}$.

By Proposition 2.4, $A=A_{\mu} \cap A_{\nu}$ is still $\omega$-closed and cofinal in $\widetilde{A}$. This guarantees that $G$ is topologically and algebraically isomorphic to the limit of the factorizing $\omega$-spectrum $\mathcal{S}_{G}=\left\{G_{\alpha}, p_{\alpha}^{\beta}, A\right\}$. Also for each $\alpha \in A$ we have two maps

$$
\mu_{\alpha}: G_{\alpha} \times G_{\alpha} \rightarrow G_{\alpha} \quad \text { and } \quad \nu_{\alpha}: G_{\alpha} \rightarrow G_{\alpha}
$$

which allow us to define:

(a) a continuous multiplication operation on $G_{\alpha}$ by letting

$$
x_{\alpha} \cdot y_{\alpha}=\mu_{\alpha}\left(x_{\alpha}, y_{\alpha}\right) \quad \text { for each }\left(x_{\alpha}, y_{\alpha}\right) \in G_{\alpha} \times G_{\alpha}
$$

(b) a continuous inversion on $G_{\alpha}$ by letting

$$
x_{\alpha}^{-1}=\nu_{\alpha}\left(x_{\alpha}\right) \quad \text { for each } x_{\alpha} \in G_{\alpha} .
$$

It is easy to see that $G_{\alpha}, \alpha \in A$, becomes a topological group with respect to these operations. Moreover, for each $\alpha \in A$ the limit projection $p_{\alpha}: G \rightarrow G_{\alpha}$ becomes a homomorphism with respect to the above defined operations.

Corollary 3.2. Let $G$ be an $\mathrm{AE}(0)$-group such that $\operatorname{dim} G \leq n$. Then $G$ is topologically and algebraically isomorphic to the limit of a factorizing $\omega$-spectrum $\mathcal{S}_{G}=\left\{G_{\alpha}, p_{\alpha}^{\beta}, A\right\}$ consisting of at most n-dimensional Polish groups $G_{\alpha}, \alpha \in A$, and 0 -soft limit homomorphisms $p_{\alpha}: G \rightarrow G_{\alpha}, \alpha \in A$. 
Proof. By Proposition 3.1, $G=\lim \mathcal{S}_{1}$, where $\mathcal{S}_{1}=\left\{G_{\alpha}, p_{\alpha}^{\beta}, A_{1}\right\}$ is a factorizing $\omega$-spectrum consisting of Polish groups and 0-soft limit homomorphisms. By [4, Theorem 1.3.10], $G=\lim \mathcal{S}_{2}$, where $\mathcal{S}_{2}=\left\{G_{\alpha}, p_{\alpha}^{\beta}, A_{2}\right\}$ is a factorizing $\omega$-spectrum consisting of at most $n$-dimensional Polish spaces. It follows directly from the proofs of Proposition 3.1 and [4, Theorem 1.3.10] that both indexing sets $A_{1}$ and $A_{2}$ can be assumed to be closed $\omega$-complete subsets of an $\omega$-complete set $B$. By Proposition 2.4, $A=A_{1} \cap A_{2}$ is still a cofinal $\omega$-closed subset of $B$. Consequently, the factorizing $\omega$-spectrum $\mathcal{S}_{G}=\left\{G_{\alpha}, p_{\alpha}^{\beta}, A\right\}$ consists of at most $n$-dimensional Polish groups and 0 -soft limit homomorphisms. It only remains to note that $G=\lim \mathcal{S}_{G}$.

Corollary 3.3. Let $\tau \geq \omega$. Every $\mathrm{AE}(0)$-group of weight $\tau \geq \omega$ is topologically and algebraically isomorphic to a closed $C$-embedded subgroup of the product $\prod\left\{G_{t}: t \in T\right\}$, where $G_{t}, t \in T$, is a Polish group and $|T|=\tau$.

Proof. Let $G$ be an $\operatorname{AE}(0)$-group of weight $\tau$. If $\tau=\omega$, then $G$ is Polish (see [4, Chapter 6$]$ ) and consequently there is nothing to prove.

If $\tau>\omega$, then by Proposition $3.1, G$ is topologically and algebraically isomorphic to the limit of a factorizing $\omega$-spectrum $\mathcal{S}_{G}=\left\{G_{t}, p_{t}^{t^{\prime}}, T\right\}$ with $|T|=\tau$. Clearly $\lim \mathcal{S}_{G}$ is isomorphic to a closed subgroup of the product $\prod\left\{G_{t}: t \in A\right\}$. Since the spectrum $\mathcal{S}_{G}$ is factorizing, it follows that $\lim \mathcal{S}_{G}$ is $C$-embedded in $\prod\left\{G_{t}: t \in T\right\}$.

In light of Corollary 3.3 it is interesting to know whether every closed $C$-embedded subgroup of the product of Polish groups is an $\mathrm{AE}(0)$-group.

Another question related to Corollary 3.3 is whether $\mathrm{AE}(0)$-groups can be characterized as closed $C$-embedded subgroups $G$ of products of Polish groups such that the projection of $G$ onto every countable face of the product is closed.

TheOREM 3.4. Let $G$ be a topological group of weight $\tau>\omega$. Then the following conditions are equivalent:

(a) $G$ is an $\mathrm{AE}(0)$-group.

(b) There exists a well ordered inverse spectrum $\mathcal{S}_{G}=\left\{G_{\alpha}, p_{\alpha}^{\alpha+1}, \tau\right\}$ with the following properties:

(1) $G_{\alpha}$ is an $\mathrm{AE}(0)$-group and $p_{\alpha}^{\alpha+1}: G_{\alpha+1} \rightarrow G_{\alpha}$ is a 0 -soft homomorphism with Polish kernel, $\alpha<\tau$.

(2) If $\beta<\tau$ is a limit ordinal, then the diagonal product

$$
\triangle\left\{p_{\alpha}^{\beta}: \alpha<\beta\right\}: G_{\beta} \rightarrow \lim \left\{G_{\alpha}, p_{\alpha}^{\alpha+1}, \alpha<\beta\right\}
$$

is a topological and algebraic isomorphism.

(3) $G$ is topologically and algebraically isomorphic to $\lim \mathcal{S}_{G}$.

(4) $G_{0}$ is a Polish group. 
Proof. (a) $\Rightarrow(\mathrm{b})$. By Corollary 3.3, we may assume that $G$ is a closed $C$-embedded subgroup of a product $\prod\left\{X_{a}: a \in A\right\},|A|=\tau$, of Polish groups $X_{a}, a \in A$. There exists a proper, functionally closed 0 -invertible map $f: Y \rightarrow \prod\left\{X_{a}: a \in A\right\}$, where $Y$ is a spectrally complete (see [4, p. 247]) realcompact space of weight $\tau$ and dimension $\operatorname{dim} Y=0$ (see [4, Proposition 6.2.13] for details). Consider the inverse image $f^{-1}(G) \subseteq Y$ of $G$ and the map $f \mid f^{-1}: f^{-1}(G) \rightarrow G$. Since $G$ is $C$-embedded in the product $\prod\left\{X_{a}: a \in A\right\}$, since $\operatorname{dim} Y=0$ and since $G$, according to (a), is an $\operatorname{AE}(0)$ space, there exists a map $g: Y \rightarrow G$ such that $g\left|f^{-1}(G)=f\right| f^{-1}(G)$.

Denote by

$$
\pi_{B}: \prod\left\{X_{a}: a \in A\right\} \rightarrow \prod\left\{X_{a}: a \in B\right\}
$$

and

$$
\pi_{C}^{B}: \prod\left\{X_{a}: a \in B\right\} \rightarrow \prod\left\{X_{a}: a \in C\right\}
$$

the natural projections onto the corresponding subproducts $(C \subseteq B \subseteq A)$. We call a subset $B \subseteq A$ admissible (cf. the proof of [4, Theorem 6.3.1]) if

$$
\pi_{B}\left(g\left(f^{-1}(x)\right)\right)=\pi_{B}(x)
$$

for each $x \in \pi_{B}^{-1}\left(\pi_{B}(G)\right)$. We need the following properties of admissible sets.

Claim 1. The union of every collection of admissible sets is admissible.

Indeed, let $\left\{B_{t}: t \in T\right\}$ be a collection of admissible sets and $B=\bigcup\left\{B_{t}\right.$ : $t \in T\}$. Let $x \in \pi_{B}^{-1}\left(\pi_{B}(G)\right)$. Clearly $x \in \pi_{B_{t}}^{-1}\left(\pi_{B_{t}}(G)\right)$ for each $t \in T$ and consequently

$$
\pi_{B_{t}}\left(g\left(f^{-1}(x)\right)\right)=\pi_{B_{t}}(x) \quad \text { for each } t \in T .
$$

Obviously, $\pi_{B}(x) \in \pi_{B}\left(g\left(f^{-1}(x)\right)\right)$ and it therefore suffices to show that the set $\pi_{B}\left(g\left(f^{-1}(x)\right)\right)$ contains only one point. Assuming that there is a point $y \in \pi_{B}\left(g\left(f^{-1}(x)\right)\right)$ such that $y \neq \pi_{B}(x)$ we conclude (remembering that $\left.B=\bigcup\left\{B_{t}: t \in T\right\}\right)$ that there must be an index $t \in T$ such that $\pi_{B_{t}}^{B}(y) \neq \pi_{B_{t}}^{B}\left(\pi_{B}(x)\right)$. But this is impossible:

$$
\pi_{B_{t}}^{B}(y) \in \pi_{B_{t}}^{B}\left(\pi_{B}\left(g\left(f^{-1}(x)\right)\right)\right)=\pi_{B_{t}}\left(g\left(f^{-1}(x)\right)\right)=\pi_{B_{t}}(x)=\pi_{B_{t}}^{B}\left(\pi_{B}(x)\right) .
$$

Claim 2. If $B \subseteq A$ is admissible, then the restriction $\pi_{B} \mid G: G \rightarrow \pi_{B}(G)$ is 0 -soft.

Let $\varphi: Z \rightarrow \pi_{B}(G)$ and $\varphi_{0}: Z_{0} \rightarrow G$ be defined on a realcompact space $Z$ with $\operatorname{dim} Z=0$ and its closed subset $Z_{0}$ respectively. Assume that $\pi_{B} \varphi_{0}=\varphi \mid Z_{0}$ and $C\left(\varphi_{0}\right)(C(G)) \subseteq C(Z) \mid Z_{0}$. We wish to construct a map 
$\phi: Z \rightarrow G$ such that $\phi \mid Z_{0}=\varphi_{0}$ and $\pi_{B} \phi=\varphi$, i.e. $\phi$ makes the diagram

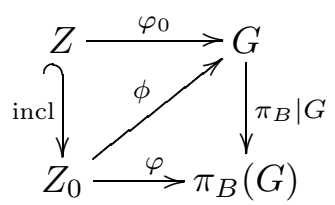

commutative. Since, according to our choice, all $X_{a}$ 's are $\mathrm{AE}(0)$-spaces (recall that each $X_{a}$ is a Polish space), so is the product $\prod\left\{X_{a}: a \in A-B\right\}$. This implies the 0 -softness of the projection $\pi_{B}$ and hence of its restriction

$$
\pi_{B} \mid \pi_{B}^{-1}\left(\pi_{B}(G)\right): \pi_{B}^{-1}\left(\pi_{B}(G)\right) \rightarrow \pi_{B}(G) .
$$

Then there exists a map $\phi^{\prime \prime}: Z \rightarrow \pi_{B}^{-1}\left(\pi_{B}(G)\right)$ such that $\phi^{\prime \prime} \mid Z_{0}=\varphi_{0}$ and $\pi_{B} \phi^{\prime \prime}=\varphi$. Since $f$ is 0 -invertible (and $\operatorname{dim} Z=0$ ), there exists a map $\phi^{\prime}$ : $Z \rightarrow Y$ such that $f \phi^{\prime}=\phi^{\prime \prime}$. Now let $\phi=g \phi^{\prime}$. Since $g\left|f^{-1}(G)=f\right| f^{-1}(G)$, we have $\varphi_{0}=\phi \mid Z_{0}$. Finally, the admissibility of $B$ implies $\varphi=\pi_{B} \phi$ as required.

Claim 3. For each countable subset $C \subseteq A$ there exists a countable admissible subset $B \subseteq A$ such that $C \subseteq B$.

Since $w(Y)=\tau$ and $\operatorname{dim} Y=0$, it follows (consult [4, Theorem 1.3.10]) that $Y$ can be represented as the limit space of a factorizing $\omega$-spectrum $\mathcal{S}_{Y}=\left\{Y_{B}, q_{C}^{B}, \exp _{\omega} A\right\}$ consisting of zero-dimensional Polish spaces $Y_{B}$, $B \in \exp _{\omega} A$, and continuous surjections $q_{C}^{B}: Y_{B} \rightarrow Y_{C}, C \subseteq B, C, B \in$ $\exp _{\omega} A$. Consider also the standard factorizing $\omega$-spectrum $\mathcal{S}_{X}=\left\{\prod\left\{X_{a}\right.\right.$ : $\left.a \in B\}, \pi_{C}^{B}, \exp _{\omega} A\right\}$ consisting of the countable subproducts of the product $\prod\left\{X_{a}: a \in A\right\}$ and the corresponding natural projections. Obviously the full product coincides with the limit of $\mathcal{S}_{X}$. One more factorizing $\omega$-spectrum arises naturally. This is the spectrum $\mathcal{S}_{G}=\left\{\pi_{B}(G), \pi_{C}^{B} \mid \pi_{B}(G), \exp _{\omega} A\right\}$ whose limit coincides with $G$.

Consider the map $f: \lim \mathcal{S}_{Y} \rightarrow \lim \mathcal{S}_{X}$. By [4, Theorem 1.3.4], there is a cofinal $\omega$-closed subset $\mathcal{T}_{f}$ of $\exp _{\omega} A$ such that for each $B \in \mathcal{T}_{f}$ there is a map $f_{B}: Y_{B} \rightarrow \prod\left\{X_{a}: a \in B\right\}$ such that $f_{B} \circ q_{B}=\pi_{B} \circ f$. Moreover, these maps form a morphism

$$
\left\{f_{B} ; B \in \mathcal{T}_{f}\right\}: \mathcal{S}_{Y} \rightarrow \mathcal{S}_{X}
$$

whose limit coincides with $f$. Since $f$ is proper and functionally closed, we may assume (see [4, Proposition 6.2.9]), considering a smaller cofinal $\omega$ closed subset of $\mathcal{T}_{f}$ if necessary, that the above morphism is bicommutative. This simply means that $q_{B} f^{-1}(K)=f_{B}^{-1}\left(\pi_{B}(K)\right)$ for any $B \in \mathcal{T}_{f}$ and any closed subset $K$ of the product $\prod\left\{X_{a}: a \in A\right\}$. 
Similarly, applying [4, Theorem 1.3.4] to the $\operatorname{map} g: \lim \mathcal{S}_{Y} \rightarrow \lim \mathcal{S}_{G}$, we obtain a cofinal $\omega$-closed subset $\mathcal{T}_{g}$ of $\exp _{\omega} A$ and the associated morphism

$$
\left\{g_{B}: Y_{B} \rightarrow \pi_{B}(G) ; B \in \mathcal{T}_{g}\right\}: \mathcal{S}_{Y} \rightarrow \mathcal{S}_{G}
$$

whose limit coincides with $g$.

By Proposition 2.4, the intersection $\mathcal{T}=\mathcal{T}_{f} \cap \mathcal{T}_{g}$ is still a cofinal $\omega$-closed subset of $\exp _{\omega} A$. Hence it suffices to show that each $B \in \mathcal{T}$ is an admissible subset of $A$. Let $x \in \pi_{B}^{-1}\left(\pi_{B}(G)\right)$. First observe that the bicommutativity of the morphism associated with $\mathcal{T}_{f}$ implies that $q_{B}\left(f^{-1}(x)\right)=f_{B}^{-1}\left(\pi_{B}(x)\right)$. Since $f_{B}$ and $g_{B}$ coincide on $f_{B}^{-1}\left(\pi_{B}(G)\right)$ we have

$$
\begin{aligned}
\pi_{B}\left(g\left(f^{-1}(x)\right)\right) & =g_{B}\left(q_{B}\left(f^{-1}(x)\right)\right)=g_{B}\left(f_{B}^{-1}\left(\pi_{B}(x)\right)\right) \\
& =f_{B}\left(f_{B}^{-1}\left(\pi_{B}(x)\right)\right)=\pi_{B}(x)
\end{aligned}
$$

as required.

Claim 4. If $C$ and $B$ are admissible subsets of $A$ and $C \subseteq B$, then the map $\pi_{C}^{B} \mid \pi_{B}(G): \pi_{B}(G) \rightarrow \pi_{C}(G)$ is 0-soft.

This property follows from Claim 2 and [4, Lemma 6.1.15].

Having all the needed properties of admissible subsets established, we proceed as follows. Since $|A|=\tau$ we can write $A=\left\{a_{\alpha}: \alpha<\tau\right\}$. By Claim 3, each $a_{\alpha} \in A$ is contained in a countable admissible subset $B_{\alpha} \subseteq A$. Let $A_{\alpha}=\bigcup\left\{B_{\beta}: \beta \leq \alpha\right\}$. We use these sets to define a transfinite inverse spectrum $\mathcal{S}=\left\{G_{\alpha}, p_{\alpha}^{\alpha+1}, \tau\right\}$ as follows. Let $G_{\alpha}=\pi_{A_{\alpha}}(G)$ and $p_{\alpha}^{\alpha+1}=$ $\pi_{A_{\alpha}}^{A_{\alpha+1}} \mid G_{\alpha+1}$ for each $\alpha<\tau$. All the required properties of the spectrum $\mathcal{S}_{G}$ are satisfied by construction.

Finally, (b) $\Rightarrow$ (a) immediately follows from [4, Proposition 6.3.4].

REMARK 3.5. Actually the 0-soft homomorphism $p_{\alpha}^{\alpha+1}: G_{\alpha+1} \rightarrow G_{\alpha}$, $\alpha<\tau$, in Theorem 3.4(b)(1) has Polish kernel in a somewhat stronger sense than in the original definition of Subsection 2.1. Namely the Polish space $P$ (from the definition), such that $G_{\alpha+1}$ admits a $C$-embedding into the product $G_{\alpha} \times P$ in such a way that $p_{\alpha}^{\alpha+1}$ coincides with the restriction of the projection $\pi_{G_{\alpha}}: G_{\alpha} \times P \rightarrow G_{\alpha}$, can be chosen to be a Polish group and the embedding of $G_{\alpha+1} \rightarrow G_{\alpha} \times P$ can be assumed to be a homomorphism. Of course this implies that $\operatorname{ker} p_{\alpha}^{\alpha+1}$, as a closed subgroup of $P$, is itself a Polish group. It would be interesting to see whether the converse of this observation is also true, i.e. if it is true that if the kernel $\operatorname{ker} p$ of a 0 -soft homomorphism $p: G \rightarrow L$ of $\operatorname{AE}(0)$-groups is Polish, then $p$ has a Polish kernel in the sense of Subsection 2.1.

Next we characterize 0 -soft homomorphisms of AE(0)-groups with Polish kernels. 
Proposition 3.6. A 0-soft homomorphism $f: G \rightarrow L$ between $\mathrm{AE}(0)$ groups has a Polish kernel if and only if there exist factorizing $\omega$-spectra $\mathcal{S}_{G}=\left\{G_{\alpha}, p_{\alpha}^{\beta}, A\right\}, \mathcal{S}_{L}=\left\{L_{\alpha}, q_{\alpha}^{\beta}, A\right\}$ consisting of Polish groups and 0-soft limit homomorphisms, and a morphism $\left\{f_{\alpha}\right\}: \mathcal{S}_{G} \rightarrow \mathcal{S}_{L}$ consisting of 0-soft homomorphisms, such that the following conditions are satisfied:

(1) $G=\lim \mathcal{S}_{G}, L=\lim \mathcal{S}_{L}$ and $f=\lim \left\{f_{\alpha}\right\}$.

(2) All limit square diagrams generated by limit projections of the spectra $\mathcal{S}_{G}$ and $\mathcal{S}_{L}$, by elements of the morphism $\left\{f_{\alpha}\right\}$ and by the map $f$ are pullback squares.

Proof. By Proposition 3.1, we may assume that both $G$ and $L$ are topologically and algebraically isomorphic to the limits of factorizing $\omega$-spectra $\mathcal{S}_{G}=\left\{G_{\alpha}, p_{\alpha}^{\beta}, A\right\}$ and $\mathcal{S}_{L}=\left\{L_{\alpha}, q_{\alpha}^{\beta}, A\right\}$, consisting of Polish groups and 0 -soft limit homomorphisms. By [4, Theorem 1.3.6], we may also assume that the map $f$ is the limit of a morphism $\left\{f_{\alpha}: G_{\alpha} \rightarrow L_{\alpha} ; A\right\}$, consisting of continuous maps $f_{\alpha}: G_{\alpha} \rightarrow L_{\alpha}$. Since $f$ itself and all the limit projections $p_{\alpha}: G \rightarrow G_{\alpha}$ and $q_{\alpha}: L \rightarrow L_{\alpha}$ are group homomorphisms, it follows easily that so is $f_{\alpha}: G_{\alpha} \rightarrow L_{\alpha}, \alpha \in A$. Finally since $f$ is 0 -soft and has a Polish kernel, it follows, by [4, Theorem 6.3.1(vi)], that all limit square diagrams

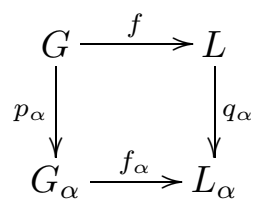

generated by the limit projections of the spectra $\mathcal{S}_{G}$ and $\mathcal{S}_{L}$, by elements of the morphism $\left\{f_{\alpha}\right\}$ and by the map $f$, are pullback squares.

In Subsection 4.1, we consider actions of $\mathrm{AE}(0)$-groups on $\mathrm{AE}(0)$-spaces. The main tool is the following statement (see [4, Theorem 8.7.1]).

Proposition 3.7. Let $\lambda: G \times X \rightarrow X$ be a continuous action of an $\mathrm{AE}(0)$-group $G$ on an $\mathrm{AE}(0)$-space $X$. Suppose that $X$ is homeomorphic to the limit space of a factorizing $\omega$-spectrum $\mathcal{S}_{X}=\left\{X_{\alpha}, p_{\alpha}^{\beta}, A\right\}$ consisting of Polish spaces and 0-soft limit projections. Suppose also that $G$ is topologically and algebraically isomorphic to the limit of a factorizing $\omega$-spectrum $\mathcal{S}_{G}=\left\{G_{\alpha}, s_{\alpha}^{\beta}, A\right\}$ consisting of Polish groups and 0-soft limit homomorphisms. Then $\lambda$ is the limit of "level actions", i.e. $\lambda=\lim \lambda_{\alpha}$, where

$$
\left\{\lambda_{\alpha}: G_{\alpha} \times X_{\alpha} \rightarrow X_{\alpha}, B\right\}: \mathcal{S}_{G}\left|B \times \mathcal{S}_{X}\right| B \rightarrow \mathcal{S}_{X} \mid B
$$

is a morphism between the spectra $\mathcal{S}_{G}\left|B \times \mathcal{S}_{X}\right| B$ and $\mathcal{S}_{X} \mid B$, and $B$ is a cofinal $\omega$-closed subset of the indexing set $A$. 


\section{Applications}

4.1. Universal $\mathrm{AE}(0)$-groups and universal actions of $\mathrm{AE}(0)$-groups. In this subsection we prove the existence of universal $\mathrm{AE}(0)$-groups of a given weight as well as the existence of a universal action of an $\mathrm{AE}(0)$-group of a given weight on a compact $\mathrm{AE}(0)$-space of the same weight.

Proposition 4.1. Let $\tau \geq \omega$. The class of $\mathrm{AE}(0)$-groups of weight $\leq \tau$ contains a universal element. More formally, every $\mathrm{AE}(0)$-group is topologically and algebraically isomorphic to a closed $C$-embedded subgroup of the power $(\operatorname{Aut}(\mathbb{Q}))^{\tau}$, where $\operatorname{Aut}(\mathbb{Q})$ denotes the group of autohomeomorphisms of the Hilbert cube $\mathbb{Q}$.

Proof. Let $G$ be an $\mathrm{AE}(0)$-group of weight $\tau$. By Corollary 3.3, $G$ is topologically and algebraically isomorphic to a closed $C$-embedded subgroup of the product $\prod\left\{G_{t}: t \in T\right\}$, where $G_{t}$ is a Polish group for each $t \in T$ and $|T|=\tau$. By Uspenskiı's theorem [15], $G_{t}$ can be identified with a closed subgroup of $\operatorname{Aut}(\mathbb{Q})$. Obviously $\prod\left\{G_{t}: t \in T\right\}$, and consequently $G$, is a closed $C$-embedded subgroup of $(\operatorname{Aut}(\mathbb{Q}))^{\tau}$.

Let $\tau>\omega$. Clearly the $\operatorname{AE}(0)$-group $(\operatorname{Aut}(\mathbb{Q}))^{\tau}$ (i.e. the $\tau$ th power of the group $\operatorname{Aut}(\mathbb{Q}))$ acts continuously on the Tikhonov cube $\mathbb{Q}^{\tau}$ via the natural action ("coordinatewise evaluation")

$$
\mathrm{ev}_{\tau}:(\operatorname{Aut}(\mathbb{Q}))^{\tau} \times \mathbb{Q}^{\tau} \rightarrow \mathbb{Q}^{\tau},
$$

defined by letting

$$
\mathrm{ev}_{\tau}\left(\left\{g_{\alpha}: \alpha<\tau\right\},\left\{q_{\alpha}: \alpha<\tau\right\}\right)=\left\{g_{\alpha}\left(q_{\alpha}\right): \alpha<\tau\right\}
$$

for each $\left(\left\{g_{\alpha}: \alpha<\tau\right\},\left\{q_{\alpha}: \alpha<\tau\right\}\right) \in(\operatorname{Aut}(\mathbb{Q}))^{\tau} \times \mathbb{Q}^{\tau}$.

TheOREM 4.2. Let $\tau>\omega$. The action $\mathrm{ev}_{\tau}:(\operatorname{Aut}(\mathbb{Q}))^{\tau} \times \mathbb{Q}^{\tau} \rightarrow \mathbb{Q}^{\tau}$ is universal in the category of actions of $\mathrm{AE}(0)$-groups of weight $\tau$ on compact $\mathrm{AE}(0)$-spaces of weight $\tau$. More formally, let $\lambda: G \times X \rightarrow X$ be a continuous action of an $\mathrm{AE}(0)$-group $G$ of weight $\tau$ on a compact $\mathrm{AE}(0)$-space $X$ of weight $\tau$. Then there exists a topological and algebraic embedding $i_{G}: G \rightarrow$ $(\text { Aut }(\mathbb{Q}))^{\tau}$ with closed image and an embedding $i_{X}: X \rightarrow \mathbb{Q}^{\tau}$ such that the following diagram commutes:

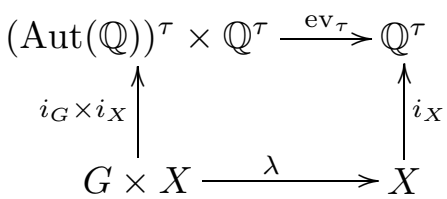

Proof. By Proposition 3.1, $G$ can be represented as the limit of a factorizing $\omega$-spectrum $\mathcal{S}_{G}=\left\{G_{\alpha}, s_{\alpha}^{\beta}, A\right\}$ consisting of Polish groups and 0-soft limit homomorphisms. Similarly, by [4, Proposition 6.3.5], $X$ can be represented as the limit space of a factorizing $\omega$-spectrum $\mathcal{S}_{X}=\left\{X_{\alpha}, p_{\alpha}^{\beta}, A\right\}$ consisting 
of metrizable compacta and 0-soft limit projections. Without loss of generality we may assume that these spectra $\mathcal{S}_{G}$ and $\mathcal{S}_{X}$ have the same indexing set $A$ and $|A|=\tau$. By Proposition 3.7, the given action $\lambda: G \times X \rightarrow X$ is the limit of level actions, i.e. $\lambda=\lim \lambda_{\alpha}$, where

$$
\left\{\lambda_{\alpha}: G_{\alpha} \times X_{\alpha} \rightarrow X_{\alpha}, B\right\}: \mathcal{S}_{G}\left|B \times \mathcal{S}_{X}\right| B \rightarrow \mathcal{S}_{X} \mid B
$$

is a morphism between the spectra $\mathcal{S}_{G}\left|B \times \mathcal{S}_{X}\right| B$ and $\mathcal{S}_{X} \mid B$, and $B$ is a cofinal $\omega$-closed subset of the indexing set $A$. We may also assume that $|B|=\tau$.

Since $G=\lim \mathcal{S}_{G} \mid B$ it follows that the diagonal product

$$
s=\triangle\left\{s_{\alpha}: G \rightarrow G_{\alpha}, \alpha \in B\right\}: G \rightarrow \prod\left\{G_{\alpha}: \alpha \in B\right\}
$$

is a topological and algebraic isomorphism with a closed image. Similarly the diagonal product

$$
p=\triangle\left\{p_{\alpha}: X \rightarrow X_{\alpha}, \alpha \in B\right\}: X \rightarrow \prod\left\{X_{\alpha}: \alpha \in B\right\}
$$

is an embedding. Consider also the product action

$$
\widetilde{\lambda}: \prod\left\{G_{\alpha}: \alpha \in B\right\} \times \prod\left\{X_{\alpha}: \alpha \in B\right\} \rightarrow \prod\left\{X_{\alpha}: \alpha \in B\right\}
$$

defined by letting

$$
\widetilde{\lambda}\left(\left\{g_{\alpha}: \alpha \in B\right\},\left\{x_{\alpha}: \alpha \in B\right\}\right)=\left\{\lambda_{\alpha}\left(g_{\alpha}, x_{\alpha}\right): \alpha \in B\right\}
$$

for each $\left(\left\{g_{\alpha}: \alpha \in B\right\},\left\{x_{\alpha}: \alpha \in B\right\}\right) \in \prod\left\{G_{\alpha}: \alpha \in B\right\} \times \prod\left\{X_{\alpha}: \alpha \in B\right\}$. Note that $\widetilde{\lambda} \circ(s \times p)=p \circ \lambda$, i.e. the following diagram is commutative:

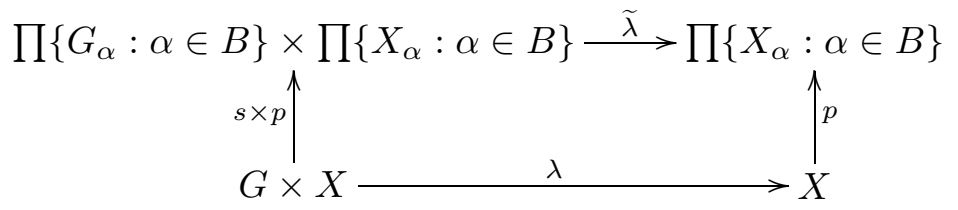

Since for each $\alpha \in B$ the group $G_{\alpha}$ is Polish, it follows by [12] (see also $[1$, Theorem 2.6.7]) that there exist a topological and algebraic embedding $j_{\alpha}: G_{\alpha} \rightarrow \operatorname{Aut}\left(\mathbb{Q}_{\alpha}\right)$ with closed image and an embedding $i_{\alpha}: X_{\alpha} \rightarrow \mathbb{Q}_{\alpha}$ (here $\mathbb{Q}_{\alpha}$ denotes a copy of the Hilbert cube $\left.\mathbb{Q}\right)$ such that $\mathrm{ev}_{\alpha} \circ\left(j_{\alpha} \times i_{\alpha}\right)=$ $i_{\alpha} \circ \lambda_{\alpha}$, i.e. the diagram

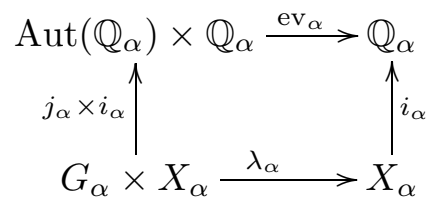

commutes for each $\alpha \in B$. Here $\operatorname{ev}_{\alpha}: \operatorname{Aut}\left(\mathbb{Q}_{\alpha}\right) \times \mathbb{Q}_{\alpha} \rightarrow \mathbb{Q}_{\alpha}$ is the evaluation action, i.e. $\operatorname{ev}_{\alpha}\left(g_{\alpha}, x_{\alpha}\right)=g_{\alpha}\left(x_{\alpha}\right)$ for each $\left(g_{\alpha}, x_{\alpha}\right) \in \operatorname{Aut}\left(\mathbb{Q}_{\alpha}\right) \times \mathbb{Q}_{\alpha}$. Let

$$
j=X\left\{j_{\alpha}: \alpha \in B\right\}: \prod\left\{G_{\alpha}: \alpha \in B\right\} \rightarrow \prod\left\{\operatorname{Aut}\left(\mathbb{Q}_{\alpha}\right): \alpha \in B\right\}
$$


and

$$
i=\chi\left\{i_{\alpha}: \alpha \in B\right\}: \prod\left\{X_{\alpha}: \alpha \in B\right\} \rightarrow \prod\left\{\mathbb{Q}_{\alpha}: \alpha \in B\right\}
$$

Finally consider the commutative diagram

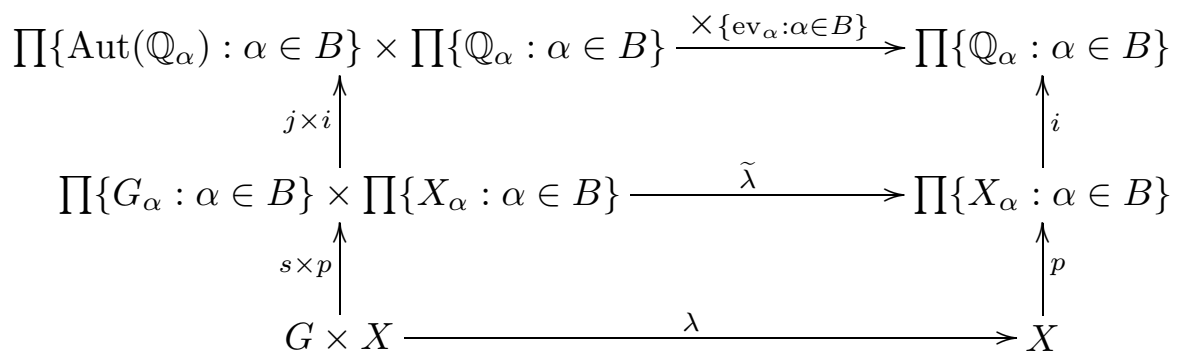

and note that since $|B|=\tau$ the upper horizontal arrow is actually the action $\mathrm{ev}_{\tau}:(\operatorname{Aut}(\mathbb{Q}))^{\tau} \times \mathbb{Q}^{\tau} \rightarrow \mathbb{Q}^{\tau}$. Clearly it suffices to let $i_{G}=(j \times i) \circ(s \times p)$ and $i_{X}=i \circ p$. This completes the proof.

It would be very interesting to prove that for an $\mathrm{AE}(0)$-group $G$ of weight $\tau>\omega$ the category of $\mathrm{AE}(0)$-spaces (of weight $\tau$ ) admitting actions of the group $G$ and their $G$-maps contains a universal object. For $\tau=\omega$ this fact has recently been proved in [9].

4.2. Closed subgroups of powers of the symmetric group $S_{\infty}$. The following result gives an embeddability criterion into the symmetric group $S_{\infty}$, the group of all bijections of $\mathbb{N}$ under the relative topology inherited from $\mathbb{N}^{\mathbb{N}}$. It is important to note [7] that there exist zero-dimensional Polish groups which cannot be embedded into $S_{\infty}$ as closed subgroups.

Theorem $4.3([1])$. Let $G$ be a Polish group. Then the following conditions are equivalent:

(i) $G$ is isomorphic to a closed subgroup of $S_{\infty}$;

(ii) $G$ admits a (countable) neighborhood basis at the identity consisting of open subgroups;

(iii) $G$ admits a (countable) basis closed under left multiplication (or a countable basis closed under right multiplication);

(iv) $G$ admits a compatible left-invariant ultrametric.

If a topological group $G$ has countable Suslin number and admits a neighborhood basis at the identity consisting of open subgroups, then $G$ is isomorphic to a topological subgroup of $S_{\infty}^{\tau}$, where $\tau=w(G)$. To see this consider left actions of $G$ on countable discrete spaces of the form $G / H$, where $H$ is an open subgroup of $G$. If $G$ is Ralkov-complete, then $G$ is 
closed in $S_{\infty}^{\tau}$. Since AE(0)-groups are Raukov-complete and have countable Suslin number, we obtain $\left({ }^{1}\right)$ the following result.

TheOREM 4.4. Let $\tau \geq 1$ be a cardinal number. The following conditions are equivalent for any topological $\mathrm{AE}(0)$-group $G$ of weight $\tau \geq \omega$ :

(i) $G$ is isomorphic to a closed subgroup of $S_{\infty}^{\tau}$;

(ii) $G$ admits a neighborhood basis at the identity consisting of open subgroups.

COROllary 4.5. Let $\tau \geq 2$. The following conditions are equivalent for any Polish group G:

(i) $G$ is topologically isomorphic to a closed subgroup of $S_{\infty}$;

(ii) $G$ is topologically isomorphic to a closed subgroup of $S_{\infty}^{\tau}$.

Corollary 4.6. There exists a zero-dimensional Polish group which is not topologically isomorphic to a closed subgroup of $S_{\infty}^{\tau}$ for any cardinal number $\tau$.

Proof. It is known [7] that there exists a zero-dimensional Polish group $G$ which is not topologically isomorphic to a closed subgroup of $S_{\infty}$. By Corollary $4.5, G$ cannot be topologically isomorphic to a closed subgroup of $S_{\infty}^{\tau}$ for any cardinal $\tau \geq 2$.

4.3. Baire isomorphisms. The main result of this subsection (Theorem 4.8) allows us to reduce in many instances (descriptive) set-theoretical considerations of general $\mathrm{AE}(0)$-groups to those for Polish groups.

Lemma 4.7. Let $f: X \rightarrow Y$ be a 0-soft homomorphism between $\mathrm{AE}(0)$ groups. Then there exists a Baire isomorphism $h: Y \times \operatorname{ker} f \rightarrow X$ such that $f \circ h=\pi_{Y}$, where $\pi_{Y}: Y \times \operatorname{ker} f \rightarrow Y$ stands for the projection onto the first coordinate.

Proof. By Proposition 2.7, there exists a Baire map $g: Y \rightarrow X$ such that $f \circ g=\operatorname{id}_{Y}$. The required Baire isomorphism $h: Y \times \operatorname{ker} f \rightarrow X$ (not a homomorphism unless $g$ is a homomorphism itself) can now be defined by letting

$$
h(y, a)=g(y) \cdot a \quad \text { for each }(y, a) \in Y \times \operatorname{ker} f,
$$

where $\cdot$ denotes the multiplication operation in $X$.

THEOREM 4.8. Every $\mathrm{AE}(0)$-group is Baire isomorphic to a product of Polish groups.

Proof. Let $X$ be an $\mathrm{AE}(0)$-group. If $w(X)=\omega$, then $X$ itself is Polish and there is nothing to prove.

$\left({ }^{1}\right)$ The proof presented here has been suggested by the referee and is significantly shorter than the original one. 
Let now $w(X)=\tau>\omega$. According to Theorem 3.4, $X$ is topologically and algebraically isomorphic to the limit of a well ordered continuous spectrum $\mathcal{S}_{X}=\left\{X_{\alpha}, p_{\alpha}^{\alpha+1}, \tau\right\}$ such that $X_{0}$ is a Polish group and the 0-soft homomorphism $p_{\alpha}^{\alpha+1}: X_{\alpha+1} \rightarrow X_{\alpha}$ has a Polish kernel for each $\alpha<\tau$.

Our goal is to prove that $X$ is Baire isomorphic to the product $X_{0} \times$ $\prod\left\{\operatorname{ker} p_{\alpha}^{\alpha+1}: \alpha<\tau\right\}$. We proceed by induction. By Lemma 4.7, there exists a Baire isomorphism $h_{1}: X_{0} \times \operatorname{ker} p_{0}^{1} \rightarrow X_{1}$ such that $p_{0}^{1} \circ h_{1}=\pi_{X_{0}}$. Suppose that for each $\alpha$, where $1 \leq \alpha<\beta<\tau$, we have already constructed a Baire isomorphism $h_{\alpha}: X_{0} \times \prod\left\{\operatorname{ker} p_{\delta}^{\delta+1}: \delta<\alpha\right\} \rightarrow X_{\alpha}$ in such a way that

1. If $\alpha+1<\beta$, then the diagram

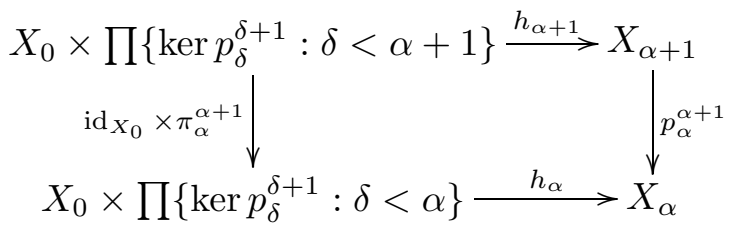

commutes, where

$$
\begin{aligned}
& \pi_{\alpha}^{\alpha+1}: \prod\left\{\operatorname{ker} p_{\delta}^{\delta+1}: \delta<\alpha+1\right\} \\
& \quad=\prod\left\{\operatorname{ker} p_{\delta}^{\delta+1}: \delta<\alpha\right\} \times X_{\alpha} \rightarrow \prod\left\{\operatorname{ker} p_{\delta}^{\delta+1}: \delta<\alpha\right\}
\end{aligned}
$$

is the natural projection.

2. $h_{\alpha}=\lim \left\{h_{\gamma}: \gamma<\alpha\right\}$ whenever $\alpha<\beta$ is a limit ordinal.

We now construct a Baire isomorphism $h_{\beta}: X_{0} \times \prod\left\{\operatorname{ker} p_{\delta}^{\delta+1}: \delta<\beta\right\} \rightarrow$ $X_{\beta}$. If $\beta$ is a limit ordinal, then we let $h_{\beta}=\lim \left\{h_{\alpha}: \alpha<\beta\right\}$. If $\beta=\alpha+1$, then consider the commutative diagram

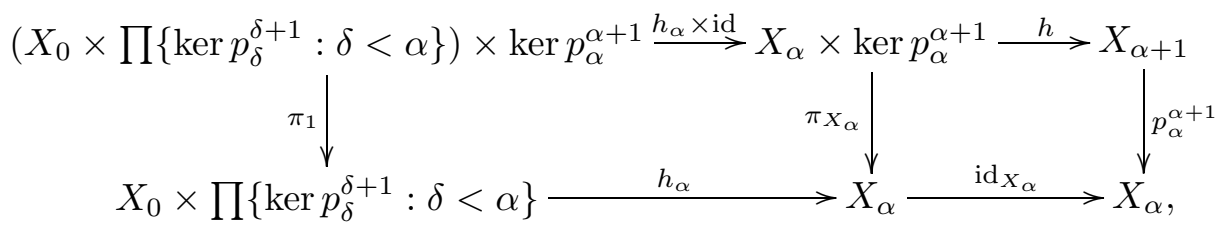

where

(a) id : $\operatorname{ker} p_{\alpha}^{\alpha+1} \rightarrow \operatorname{ker} p_{\alpha}^{\alpha+1}$ stands for the identity map;

(b)

$$
\pi_{1}:\left(X_{0} \times \prod\left\{\operatorname{ker} p_{\delta}^{\delta+1}: \delta<\alpha\right\}\right) \times \operatorname{ker} p_{\alpha}^{\alpha+1} \rightarrow X_{0} \times \prod\left\{\operatorname{ker} p_{\delta}^{\delta+1}: \delta<\alpha\right\}
$$

denotes the projection onto the first coordinate and

(c) $h: X_{\alpha} \times \operatorname{ker} p_{\alpha}^{\alpha+1} \rightarrow X_{\alpha+1}$ is a Baire isomorphism whose existence is guaranteed by Lemma 4.7 .

The required Baire isomorphism $h_{\alpha+1}: X_{0} \times \prod\left\{\operatorname{ker} p_{\delta}^{\delta+1}: \delta<\alpha+1\right\} \rightarrow$ $X_{\alpha+1}$ can now be defined as the composition $h_{\alpha+1}=h \circ\left(h_{\alpha} \times\right.$ id). This 
completes the induction and finishes the construction of Baire isomorphisms $h_{\alpha}, \alpha<\tau$. It is now easy to see that

$$
h=\lim \left\{h_{\alpha}: \alpha<\tau\right\}: X_{0} \times \prod\left\{\operatorname{ker} p_{\alpha}^{\alpha+1}: \alpha<\tau\right\} \rightarrow X
$$

is the required Baire isomorphism. Note here that $X_{0}$ as well as $\operatorname{ker} p_{\alpha}^{\alpha+1}$, $\alpha<\tau$, are Polish groups. The proof is complete.

The author is grateful to the referee for valuable comments and suggestions.

\section{References}

[1] H. Becker and A. Kechris, The Descriptive Set Theory of Polish Group Actions, London Math. Soc. Lecture Note Ser. 232, Cambridge Univ. Press, Cambridge, 1996.

[2] M. Bell and A. Chigogidze, Topological groups homeomorphic to products of discrete spaces, Topology Appl. 53 (1993), 67-73.

[3] A. Chigogidze, On spaces Baire isomorphic to the powers of the real line, Topology Appl. 107 (2000), 57-66.

[4] - Inverse Spectra, North-Holland, Amsterdam, 1996.

[5] —, Compact groups and absolute extensors, Topology Proc. 22 (1997), 63-70.

[6] - Topological characterization of torus groups, Topology Appl. 94 (1999), 13-25.

[7] R. Dougherty, Examples of non-shy sets, Fund. Math. 144 (1994), 73-88.

[8] R. Haydon, On a problem of Petczyński: Milutin spaces, Dugundji spaces and AE(0-dim), Studia Math. 52 (1974), 23-31.

[9] G. Hjorth, A universal Polish G-space, Topology Appl. 91 (1999), 141-150.

[10] K. H. Hofmann and S. A. Morris, The Structure of Compact Groups, de Gruyter Stud. Math. 25, de Gruyter, Berlin, 1998.

[11] A. Kechris, Classical Descriptive Set Theory, Springer, New York, 1995.

[12] M. Megrelishvili, Free topological G-spaces, New Zealand J. Math. 125 (1996), 59-72.

[13] V. Pestov, Topological groups: where to from here?, preprint, 1999.

[14] L. S. Pontryagin, Topological Groups, Princeton Univ. Press, Princeton, NJ, 1946.

[15] V. V. Uspenskǐ̌, A universal topological group with a countable base, Funct. Anal. Appl. 20 (1986), 160-161.

Department of Mathematics and Statistics

University of Saskatchewan

McLean Hall, 106 Wiggins Road

Saskatoon, SK, S7N 5E6, Canada

E-mail: chigogid@math.usask.ca

Received 4 January 2000;

in revised form 11 June 2000 